\title{
Image Processing of Automatic Hologram Synthesizer for Medical Use
}

\author{
Tomohiko HATTORI, Sadayuki SAKUMA, \\ Mitsuru IKEDA, Riyahi-Alam NADER \\ Nagoya University School of Medicine, Department of Radiology \\ 65 Tsurumai-cho, Showa-ku, Nagoya 466, Japan
}

Received May 22, 1992.

\section{ABSTRACT}

An image processing system for providing original images for synthesizing multiplex holograms is developed. This system reconstructs $3 \mathrm{D}$ (three dimensional) surface rendering images, two surfaces rendering images and/or band selected Computed Tomography values' projection images of internal organs and/or bones of a patient from a series of tomograms such as CT (Computed Tomography). Image processing includes interpolation, enhancement, extraction of diseased parts, selection of axis of projection, and compensation of distortions. This research note presents the features of this system, and the results of new type images with conventional images.

\section{Introduction}

Medical conventional input images for multiplex holographic display are surface reconstruction data from $X-$ ray CT, MRI (Magnetic Resonance Image) ${ }^{1)-4}$. The reconstruction data have only a surface shape's information of internal organs and/or bones of a patient. In this research note projection images for the input image composed of the band selected CT values of a series of the sectional data are proposed and the features of this system are presented.

\section{Objectives of Image Processing}

Image data obtained by medical systems like $\mathrm{X}$-ray $\mathrm{CT}$, and MRI (later in this research note, "CT" calls these systems in general) are collections of sectional images of patient's body. These slice data have 3D information, so they are sufficient to make 3D images. The purpose of the image processing system is to synthesize $3 \mathrm{D}$ images which make holograms from original medical images using a conventional surface reconstruction technique and our band selected CT values' projection technique. In addition, various additional processings which are necessary for medical use (for example, extraction of diseased parts) and for making holograms (for example, compensation of distortions) can be carried out.

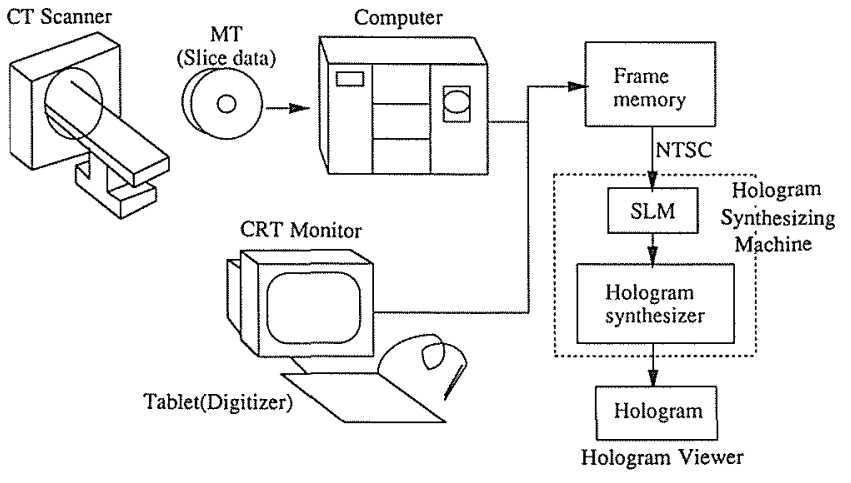

Fig. 1 Conceptual image of system configuration

\subsection{System configuration}

Fig. 1 shows the conceptual image of system configuration.

The main computer is Data General's MV/15000, and it has $32 \mathrm{MB}$ main memory and about $1 \mathrm{~GB}$ disk storage. A 19 inch color display $(1280 \times 1024$ pixels $)$ and a digitizer are used to extract concerned parts (for example, bones, diseased organs, etc.) and to monitor the slices or synthesized 3D images. 
Controller Main body Film processing machine

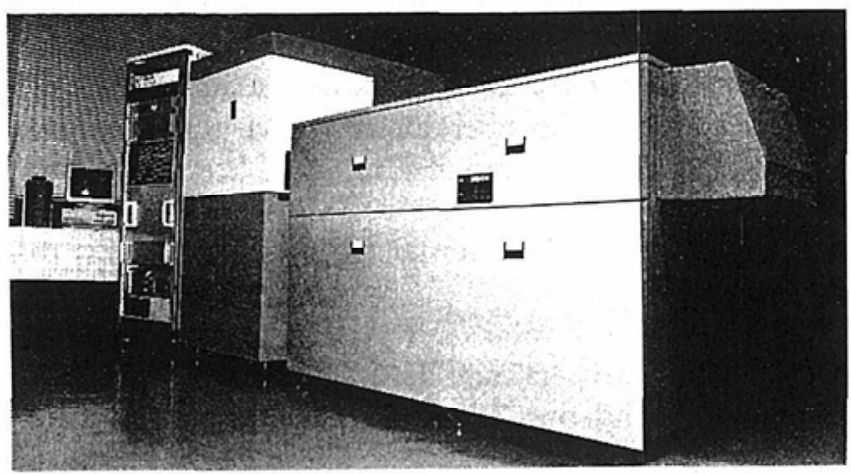

Fig. 2 Hologram synthesizing machine

The hologram synthesizing machine consists of a main body, a controller and a film processing machine as shown in Fig. 2. Optical components such as liquid crystal SLM (Spatial Light Modulator), projection optical system, ref-

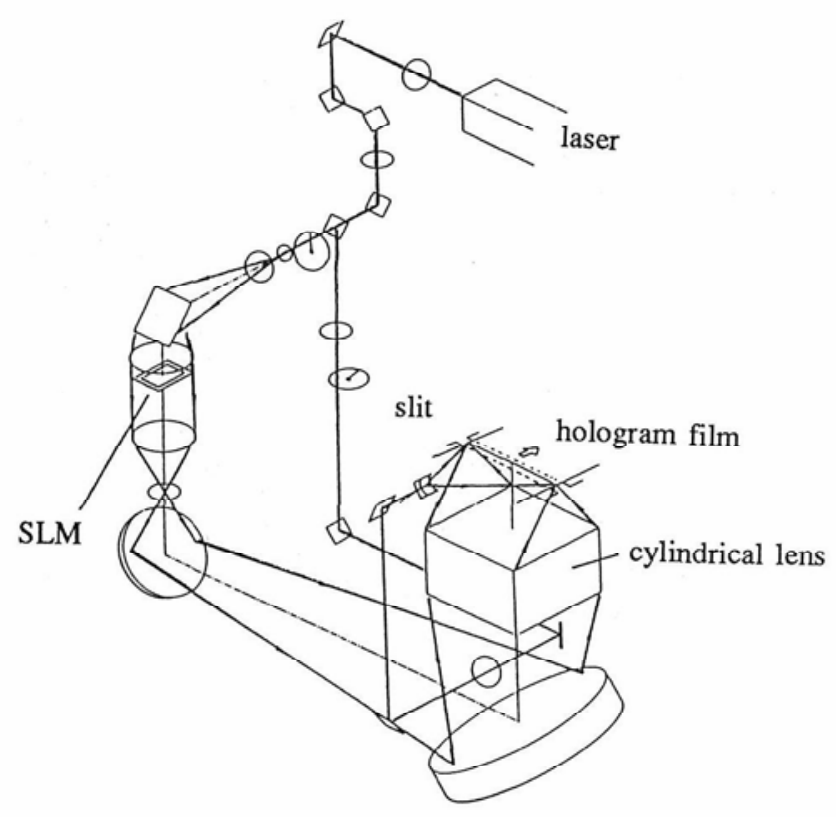

Fig. 3 Optical system of the hologram synthesizing machine

erence light optical system and hologram film supporting and transporting mechanism are fixed in the main body. The optical system is arranged three-dimensionally as shown in Fig. 3. The main body is controlled by the controller as follows. Once hologram synthesis starts, the exposure and the transportation of hologram film are made one after another. The film is transferred $0.5 \mathrm{~mm}$ at one time. Seven element holograms are exposed for each of 360 original images which produced by the main computer and translated to the SLM. So one multiplex holo- gram consists of 2520 elementary holograms. If all elementary holograms are exposed, the film is cut with a knife and sent to the film processing machine. The film processing machine is connected with the hologram synthesizing machine and receives exposed film from synthesizing machine and develops them automatically.

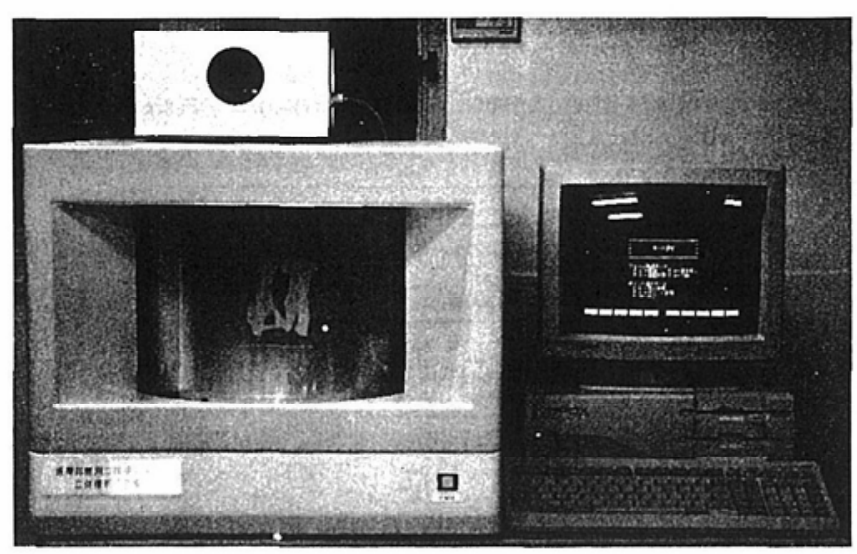

Fig. 4 Hologram viewer

The hologram viewer consists of a film supporting device and a three-dimensional measuring device, a personal computer and a light source as shown in Fig. 4. The viewing zone is 120 degrees horizontally. A LED pointer which can move freely in space in the hologram is the three-dimensional measuring device controlled by the personal computer. The position of the pointer can be meausured as the three-dimensional coordinate of the holographic image. The three-dimensional measuring unit is arranged not to interfere the hologram transporting device and light source. The light source is composed of a fiber guided metal halide lamp because of its high efficiency, long life and much visible light percentage.

\subsection{Features of image processing}

Slice data from CT scanner are fed into the computer with MT (Magnetic Tape). The image processing system will synthesize 360 surroundings image at an interval of 1 degree by surface reconstruction or band selected projection.

The image processing includes the following features:

* Regularization of various modes such as direction, aspect ratio, etc.

* Window level adjustment

* Extraction of concerned parts by man-machine interface and/or automatic operation

* Combination of two surface reconstruction data

* Compensation of distortions

Matrix size of each slice can be $128 \times 128,256 \times 256$, or $512 \times 512$. Different size of slice data must be regularized 
to one of these sizes. All image processing can be performed in one or two hours when matrix size is $256 \times$ 256 , and the extraction is done automatically by CT values.

Each image is converted to NTSC (National Television System Committee) signal through a frame memory, and the signal drives SLM in the hologram synthesizer.

\section{Flow of Image Processing}

Fig. 5 shows the general step of image processing.

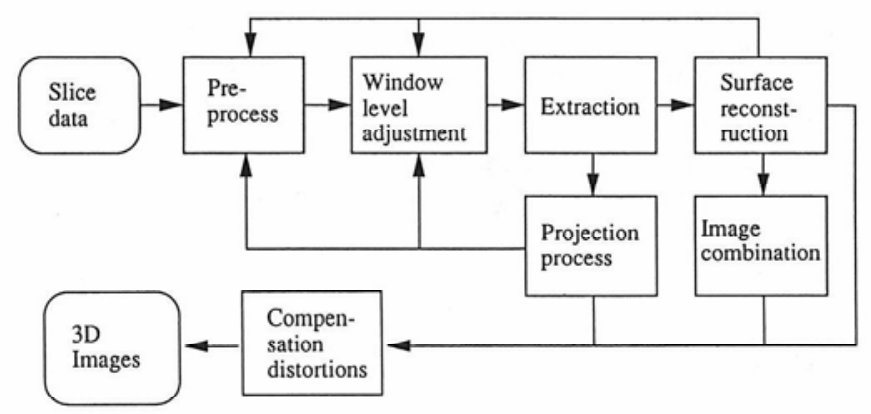

Fig. 5 Flow of Image Processing

\subsection{Pre-processing (Conversion of data format and mode)}

Original slice data are fed in by means of MT. As MT format on our system adopts the format which has been standardized by Japan Industrial Association of Radiation Apparatus, but unfortunately most CT scanners do not support this format and many uncompatible formats are used. So, these formats are converted in this stage. Additionally, some pre-processing modifies the original data. There are a variety of modes in data, such as direction, slice pitch, and matrix size. These can be regularized in advance.

\subsection{Window level adjustment}

To make the image of concerned parts as clear as possible, window level is adjustable. Two parameters which are the width of CT values and its middle value decide visible range. Only CT values inside the range are gray-scaled. Concerned parts can be enhanced by window level adjustment.

\subsection{Extraction}

The extraction of diseased parts is carried out by two types of criterion for processing of surface reconstruction, CT value and area tracing. In X-ray CT, CT value of bone is distinctly high in comparison with other parts, so area of bones can be extracted easily to select pixels whose values are higher than a specified value. This method has a merit that the processing can be done automatically but is not effective to separate organs whose CT values are near to the other parts. In this case, in addition to CT value's criterion, the operator can trace the concerned parts using digitizer, monitoring any slice image on a CRT display. With combination of CT value and tracing, particular parts of the internal organs such as brain can be extracted.

In X-ray CT, CT values correspond to many type tissues of a body, so particular tissues can be extracted easily to select a band of CT values between two specified values. This method has a merit that the processing can be done automatically and is effective to produce a high quality projection image.

3.4 Surface reconstruction and combination of two surfaces

Image processing creates 3D images in the computer, then creates projection images onto specific planes (cf Fig. 6, Fig. 7), using the surface reconstruction tech-

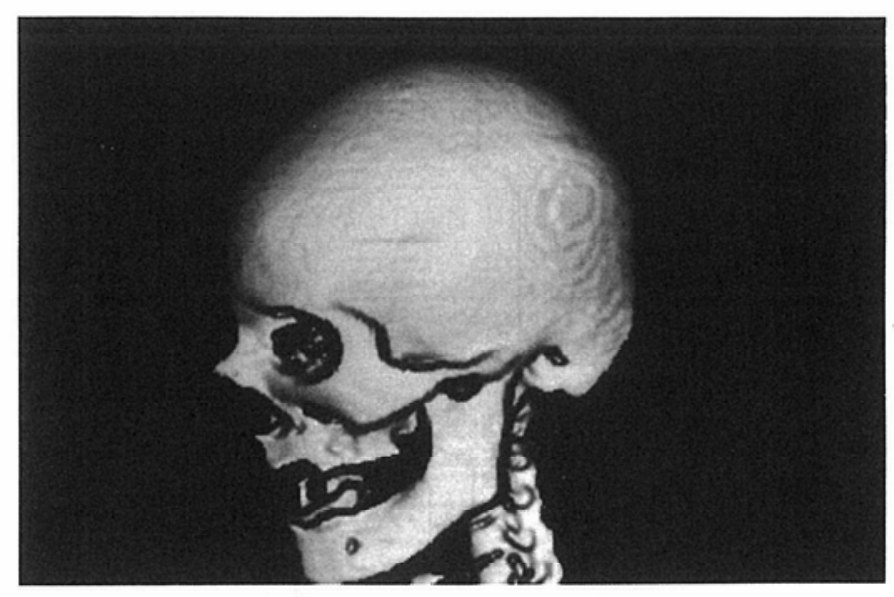

Fig. 6 Surface reconstruction image of a skull's bone

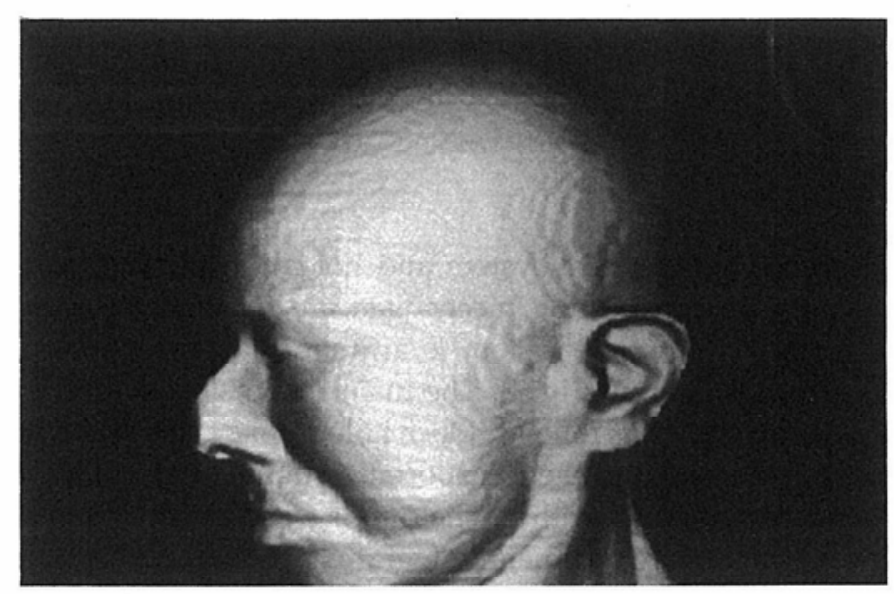

Fig. 7 Surface reconstruction image of a skull's skin 
nique. Two shading parameters (visual angle and distance) are used, and they are adjustable experimentally. Two surface reconstruction images produced from a series of slice data can be combined with each other (for example, the surface of body and the surface of bones) (cf Fig. 8). The combination ratio is variable (cf Fig. 9).

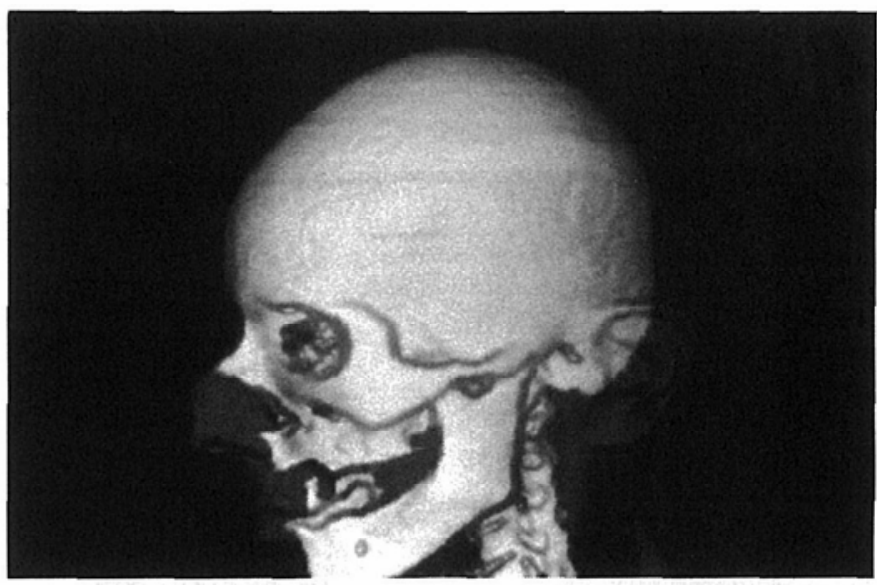

Fig. 8 Two surface reconstruction image of a skull's bone and skin

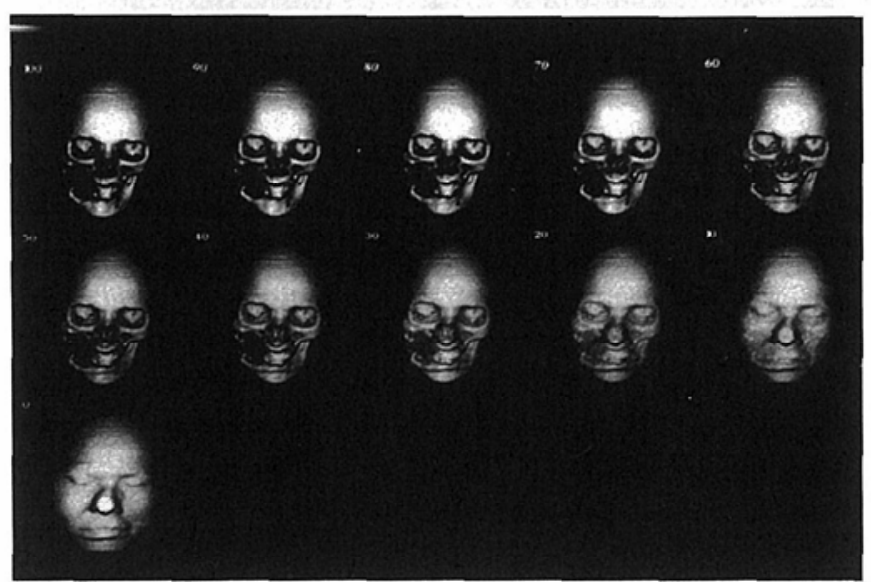

Fig. 9 Two surface reconstruction images of 11 combination ratios

\subsection{Projection of band selected CT values}

Image processing creates projection images in computer, then creates projection images by integrating the band selected CT values. The band width is narrower than the conventional X-ray images. Hence, high contrast of the specific tissue's image is available.

\subsection{Compensation of distortions}

There are two types of compensation of distortions. One is the correction of static distortion of hologram synthesis due to the change of line sight between making original images and reconstructing holograms. This type of compensation is hologram specific. Without this compensation $3 \mathrm{D}$ image is synthesized with the assumption that view point is at infinity. But actually, observers will observe the hologram from a finite distance, normally one or a few meters. As a result, 3D image will be distorted, that is, further parts of image will be larger than nearer parts. As shown in Fig. 10, the size of $O A_{1}$ is $\mathrm{OA}_{2} \cos \theta$ when $\mathrm{O}$ is a substantial center of synthetic image of hologram, $\theta$ is an angle between $O H_{\alpha}$ and $A_{l} H_{\alpha^{\prime}} H_{\alpha}$ is a point of the hologram's surface which is perspective the image point $A_{1}$ by an observer. Therefore the compensation of the distortion is to compose a perspective angle image of an elementary hologram by several other perspective angle $\theta$ images, when $\theta=\tan ^{-1}(L / r), r$ is the radius of the multiplex hologram, $L$ is the holizontal distance between the surface of the holographic image and the point $O$.

The other type of compensation is the gamma correc-

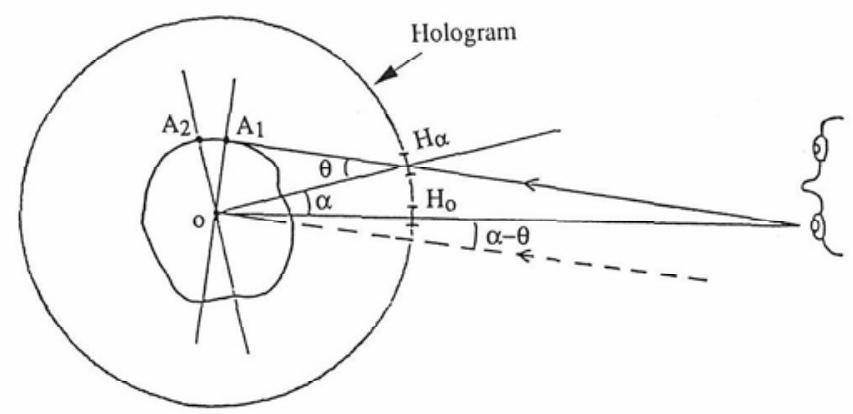

Fig. 10 Static distortion of hologram synthesis

tion for the liquid crystal SLM. This type of distortion comes from the variety of origins and their combination appears, then the accurate quantitative analysis is difficult. So, the image processing software prepares several gamma correction programs, and the best parameter can be selected experimentally from the synthesized hologram.

Synthesized 3D images are fed into the hologram synthesizer through a frame memory from 0 degree to 360 . degrees by a step of 1 degree. Before the first image, the brightest image will be sent for brightness adjustment of hologram synthesizer.

\section{Discussion}

Obtained holograms display 3D X-ray images of a patient, but the images in general are of low quality. This is because the contrast of the medical X-ray image is usually low. Fig. 11 shows the simple projection image obtained from X-ray CT slice data of a human head. The projection image is poor to produce a hologram too. 


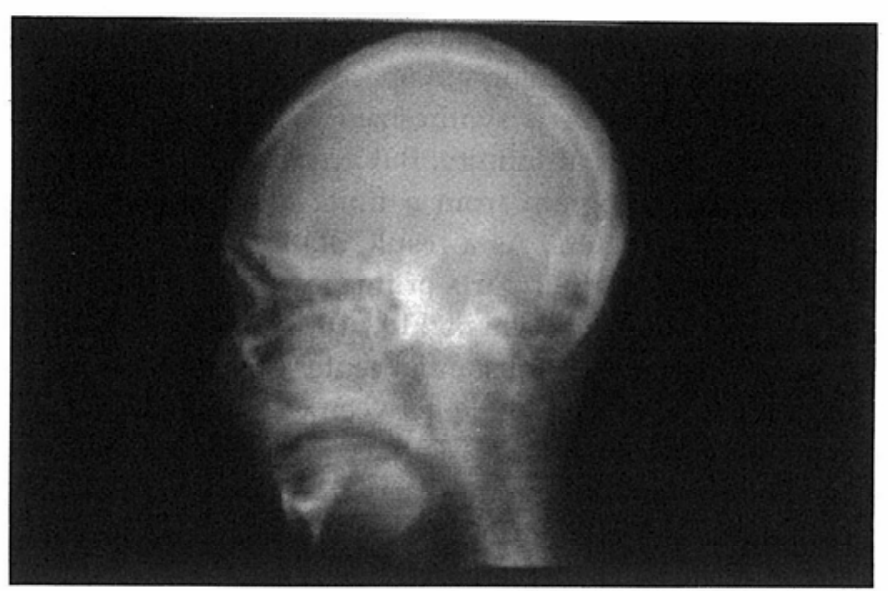

Fig. 11 Simple projection image of a skull

To obtain better images, a computer processing of the original images seems effective. For that purpose, a prototype of computer-assisted hologram synthesizing system is constructed.

Fig. 5 shows a block diagram of the system. Original images from two kinds of medical imaging modalities taken around the patient are fed in a computer, and are processed with necessary operations such as enhancement, distortion correction and interpolation.

As for conventional surface reconstruction method, the obtained images have no information except the shape of the surface of specific tissues. Such images of Fig. 6 and Fig. 7 are poor to perceive the inside of the tissue and its CT values.

In Fig. 12 the image is a projection image composed of only soft tissues' CT values. The tissue can be perceptible and the gray level corresponds to the $\mathrm{CT}$ value of the soft

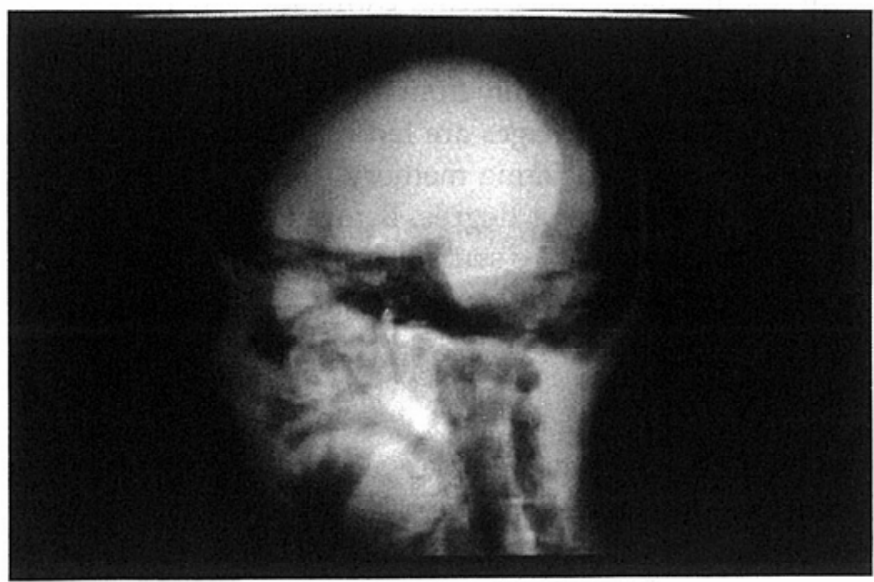

Fig. 12 Projection image of a skull's soft tissue tissue. Using conventional method regarding radiograph, the image can be obtained only from a patient's body surgically gotten bone out. Therefore such projection image composed from slice data of band selected CT values is a new type X-ray image. Moreover, the image can be perceptible as a 3D image such as multiplex hologram or any other stereoscopic displays.

In Fig. 13 whole bone structure can be perceptible and the image is dramatically sharp. The image is composed of only bone CT values.

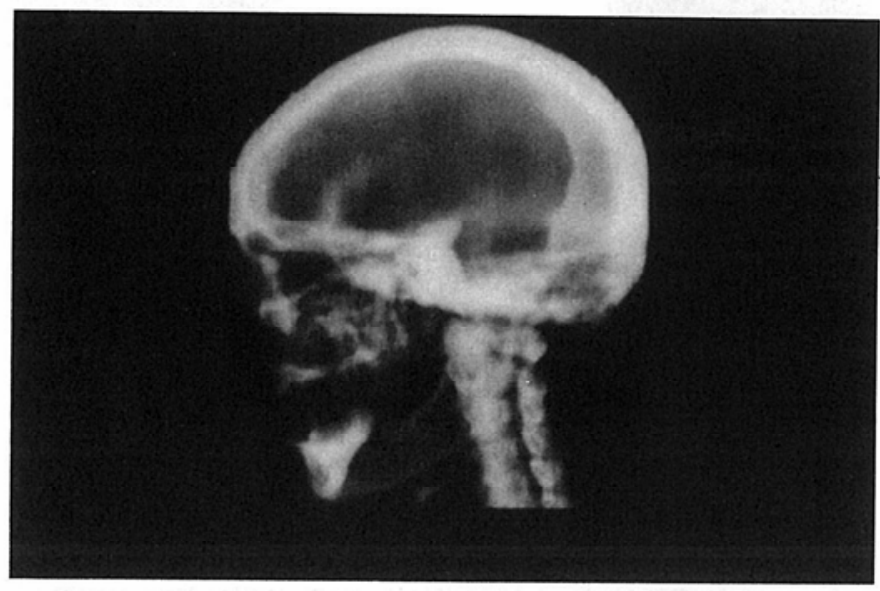

Fig. 13 Projection image of a skull's bone

All images in this paper are produced using our system from only a series of X-ray CT sectional images. Hence, we can make several multiplex holograms from a series of CT sectional images of a patient. In some case, true 3D image such as hologram doesn't need shading that is a kind of imaging processing for computer graphics. CT number is more important information than the shading for medicine. The true 3D image can directly reflect the $\mathrm{CT}$ numbers on its image.

The band selected projection 3D image composed of MRI slice data is now under experiment for research, because the image is too complex to understand the mean of parameters from a medical viewpoint.

\section{Conclusions}

The projection images for synthesizing multiplex holograms described here have whole information regarding specific tissues' CT values. The images belong to a new category of X-ray image. Hence, our automatic hologram synthesizer for medical is are able to make many type multiplex holograms for many medical requirements from a series of CT sectional images of a patient.

\section{Acknowledgements}

The authors wish to thank Masane Suzuki of the Art Department at Nagoya College of Art and Design and 
Toshikazu Misaki of Nippon-Data General Corporation for their useful discussions. This study has been carried out in the Technology Research Association of Medical and Welfare Apparatus under the sponsorship of the New Energy and Industrial Technology Development Organization as one of the subjects of the National Research and Development Program for Medical and Welfare Apparatus, Ministry of International Trade and Industry, Japan and under the sponsorship of Ministry of Education, Japan.

\section{References}

(1) Tsujiuchi J., Development of automatic hologram synthesizer for medical use I. General description, Proc. of the SPIE/IS\&T Symposium on Electronic
Imaging Science and Technology, Vol 1667 Feb. 1992

(2) Suzuki M. and Saito T., Development of automatic hologram synthesizer for medical use II. Hologram synthesizing machine and viewer, Proc. of the SPIE/ IS\&T Symposium on Electronic Imaging Science and Technology, Vol 1667 Feb. 1992

(3) Yamamoto T., Misaki T. and Kato T., Development of automatic hologram synthesizer for Medical use III. Image processing for original medical images, Proc. of the SPIE/IS\&T Symposium on Electronic Imaging Science and Technology, Vol 1667 Feb. 1992

(4) Kasazumi K., Kato M. and Uno M., et. al, Development of automatic hologram synthesizer for medical use IV. Liquid-crystal spatial light modulator, Proc. of SPIE/IS\&T Symposium on Electronic Imaging Science and Technology, Vol 1667 Feb. 1992 